\title{
LA CALIGRAFÍA MINADA DE VERÓNICA VIOLA FISHER
}

\author{
VERÓNICA VIOLA FISHER'S MINED CALLIGRAPHY
}

\author{
Victoria Cóccaro \\ Literatura, Poesía, Teoría y crítica literaria. \\ Instituto de Literatura Hispanoamericana - Universidad de Buenos Aires - CONICET. \\ Argentina. \\ victoriacoc@gmail.com
}

\begin{abstract}
Resumen: El poemario Hacer sapito de Verónica Viola Fisher publicado en 1995 se enmarca en el horizonte de una época en que las prácticas artísticas interrogan o ponen de manifiesto los modos en que el poder/discurso, siguiendo perspectivas foucaulteanas, produce los cuerpos. Nos interesa reflexionar sobre los modos en que Hacer sapito dialoga con la caída de la representación de un cuerpo estable y definible, aborda la cuestión de la infancia y la identidad de la mujer y se interroga por la posibilidad de escribir sobre lo femenino con una lengua que no le pertenece, la lengua del padre.
\end{abstract}

Palabras clave: Cuerpo, lengua, poesía argentina contemporánea, feminismo.

\begin{abstract}
Veronica Viola Fisher's book of poems, Hacer sapito, published in 1995, is framed in the horizon of an era in which artistic practices interrogate or reveal the ways in which power/discourse, following Foucauldian perspectives, produces bodies. We are interested in reflecting on the ways in which Hacer sapito talks with the fall of the representation of a stable and definable body, addresses the issue of childhood and the identity of women, and wonders about the possibility of writing about the feminine with a language that does not belong to her, the language of the "father".
\end{abstract}

Keywords: Body, language, contemporary Argentinean poetry, feminism.

Recibido: 7/08/2018. Aceptado: 14/05/19. 
Cuando una frase se hace verso deja el discurso $y$ entra en el transcurso, en el incurso, en el percurso iy hasta el recurso! pero no hace discurso. Hace otra cosa

María Salgado (2016)

\section{$\mathrm{E}$} s indiscutible que la visibilización de cuerpos violentados es una constante en las producciones estéticas en el retorno de las democracias, cuando la pregunta por los cuerpos desaparecidos se convierte en un síntoma de la cultura del Cono Sur. Como ha señalado Ana Longoni (2014) y articulado junto a la Red Conceptualismos del Sur en una exposición realizada en Madrid en 2012 y en Buenos Aires en 2014, se trata de cuerpos que pierden su forma humana. Así, por un lado, funcionan como documento de los efectos de la violencia infligida por las dictaduras militares pero, por otro, esa metamorfosis los hace emerger como soportes políticos y artísticos donde se articulan experiencias de resistencia. El cuerpo, visibilizado mediante figuras no humanas y/o cuestionando categorías humanistas (sujeto, persona, identidad, género, etc.), funciona como concepto que articula y da sentido a prácticas artísticas y discursos críticos desde la década del 80 en adelante. Entre la multiplicidad de realizaciones pueden recordarse los retratos fotográficos de Liliana Maresca con objetos-prótesis creados por ella que intervenían su imagen corporal, tomados por Marcos López; mientras que en la escritura el poema "Cadáveres" de Néstor Perlongher, publicado por primera vez en 1984 y luego en su libro Alambres en 1986, marca un horizonte de época en esta dirección.

En 1995, Verónica Viola Fisher publica Hacer sapito por Nusud, editorial que junto a la revista 18 whiskies conformó el espacio donde emergieron los primeros nombres de los poetas de la década del 90. Hacer sapito es una serie de poemas que trabaja con repeticiones, encabalgamientos y una sintaxis rota que desacata normativas hegemónicas para mostrar los modos de inscripción de la violencia en los cuerpos, en particular, la que se inscribe en los cuerpos de las mujeres al ser nombradas dentro de un discurso masculino patriarcal. Por un lado, entonces, este poemario se enmarca en el horizonte de una época en la que las prácticas artísticas, a partir del retorno de las democracias, interrogan o ponen de manifiesto los 
modos en que el poder/discurso, siguiendo perspectivas foucaulteanas, produce los cuerpos. Por otro, anuncia la inestabilidad en la figuración del cuerpo en textos escritos por mujeres, movimiento que se terminará de conformar en la segunda mitad de la década del 9o, donde lo femenino aparecerá "más como pregunta y como gesto disruptivo que como categoría que se da por sentada” (tal como sostiene Marina Yuszczuk (2011) y también advierte Laura Arnés (2016), sobre la carencia de identidades fijas en la figuración de pasiones lesbianas), una inestabilidad que se despliega en contraposición al "discurso afirmativo sobre lo femenino" que aparecía en la década del 80 en, por ejemplo, obras como Eroica de Diana Bellessi, La luz en la ventana o El mundo incompleto de Irene Gruss y Madam de Mirta Rosenberg.

Así como Hacer sapito dialoga con la caída de la representación de un cuerpo estable y definible al hacerlo aparecer mediante el fragmento, el corte y la sustracción, es también en la brevedad de los versos y en los encabalgamientos que puedo arriesgar una lectura de la caligrafía de Viola Fisher. El término caligrafía lo tomo prestado de Ana Porrúa (2011), quien propone que para atender a los segmentos mínimos y detalles del lenguaje en su materialidad es necesario no circunscribirse a la noción de escritura. Define a la caligrafía siguiendo a Barthes: como el gesto que adquiere la mano al tomar una herramienta y dejar una marca. La pregunta, entonces, sería ¿qué le hace Viola Fisher al lenguaje cuando escribe?, ¿de qué lenguaje se trata?, ¿̇con qué fragmentos cuenta y cuáles produce?

Hacer sapito pone en escena dos voces, la de la hija y la del padre, las cuales diagraman los puntos de una extraña "novela familiar" (Rubio, 1997) desde, podríamos esbozar, el nacimiento de la hija ("De mamá tuve un cordón umbilical", dice un verso de los primeros poemas) hasta la muerte del padre ("muerta de padre", es el primer verso del anteúltimo poema), pasando por otros momentos como, por ejemplo, cierta etapa educativa vinculada con la formación musical ("tampoco quiso ser/ instrumentista/ yo pagué profesores/ compré cuerdas teclas/ miel para su garganta/ por si debía cantar pero nada/ más que silencio/ iTijeras! grité”) donde ya se anuncia la disonancia como opción estética de Viola Fisher.

Una "novela" cuya narración está hecha de cuadros aislados pero cada uno es una escena clave para la configuración de la identidad de la mujer como "hija" dentro de la institución familiar (en la casa, con sus muñecas, 
tocando el piano) y todas se ven constantemente atravesadas por la violencia. Estas escenas suspendidas se nombran, en su mayoría, en presente (aun los poemas que comienzan en pasado, terminan en presente), porque ese es el material del que se dispone: todo ese conglomerado que conforma el habla, la lengua en uso, indefectiblemente en presente. Así, mientras muestran los restos de una novela familiar, ambas voces se disputan el uso de la lengua. Los poemas avanzan como campo de batalla entre una voz que posee todas las palabras, la del padre (que en uno de los poemas pronuncia: "la lengua yo"), y otra que no las tendría, la de la hija, que, entonces, si habla es porque ha robado: "en mi tinta hunde su mano", dirá la voz del padre, y en otro poema: "Mi hija se burla/ de mí/ miren cómo me saca/ la lengua yo/ su propio y único/ padre burlado?”. O bien, se la califica como ruido, sollozo o chillido: "la lengua/ se la tragó una noche/ por llorar de más"; "Como vos papito/ me chilló". La problemática que exponen los poemas es que la hija usa una lengua cuya propiedad no le está permitida, porque es del padre. En 1988, Diana Bellessi había escrito en un ensayo publicado en el Diario de poesía que la escritura de las mujeres se enfrenta al desafío de trabajar "con un discurso prestado: el del productor". No es casual que este sea uno de los atributos del padre en Hacer sapito: "nada resulta más agradable para él que la protagonista solo hable de su padre el productor" dirá la hija, mientras que el padre se reconoce: "Yo soy el productor casi único". Entonces, ¿̇cómo escribir?

En su análisis sobre cuatro poetas argentinas que escribieron y publicaron desde la década del 80 en adelante (Diana Bellessi, Tamara Kamenszain, Irene Gruss, María del Carmen Colombo), Alicia Genovese señala que ellas inauguraron un espacio cuando "reescriben el discurso social que ha expulsado a las mujeres del lugar de quien habla" (1998: 16), creando figuras que transforman y se reapropian de los espacios y roles inscriptos en sus cuerpos, construyendo así "decires poéticos femeninos" donde la mujer se proyecta en "una voz encubierta" (1998: 15) y obtiene un espacio en el que su subjetividad se imprime. Así, la escritura se convierte en una fortaleza o se combina, en muchos casos, con una fantasía de aislamiento.

Hacer sapito, lejos de pensarse como la fortaleza del espacio privado de la intimidad o un modo de irse a una isla desierta ("yo quisiera, como Gauguin, largar todo e irme, [...] irme a escribir a alguna isla", rezaba un 
poema de Irene Gruss de 1987), pone de manifiesto a la escritura como operación sobre la lengua en tanto espacio público. Se diferencia, entonces, de aquella serie de poetas porque expone a la voz en una contienda permanente. Como sugieren Marina Yuszczuk (2011) y Ana Porrúa (2011), Viola Fisher corta con esta tradición porque no crea una lengua "propia" de la subjetividad femenina, ya que pareciera no haber un espacio resguardado y elidido para la voz de la hija. Los poemas, antes que construir una voz propia, exhiben cuál es el material del que disponen y, así, exhiben la lengua de la que dispondrían para tal fin, confirmando que no existe "un sistema de signos absolutamente subjetivo para agitaciones subjetivas" (Adorno 2004, 19). Al trabajar conscientemente con el material, este poemario arma una puesta en escena de dos voces que se enfrentan y configuran: la de la hija, allí, diseñará posibles estrategias para desactivar los nombres y órdenes impuestos por el régimen patriarcal y, sobre esas ruinas, ensayar una lengua.

En una charla que un poeta contemporáneo a Viola Fisher, Martín Gambarotta (2006), da en el 2004, se pregunta si la poesía no es la voz de una tribu y si el habla no sería, entonces, la materia prima del poema. ¿Cuál es la tribu de Viola Fisher? Y ¿̇cuál es el habla que hace de materia prima a Hacer sapito?, ¿cómo aparece la lengua, ese "objeto en el que se inscribe el poder desde toda la eternidad humana” (Barthes, 2004), en la escritura?

\section{El habla como materia prima}

Las palabras no son jamás locas (a lo sumo son perversas), es la sintaxis la que es loca: ¿no es al nivel de la frase que el sujeto busca su lugar -y no lo encuentra- o encuentra un lugar falso que le es impuesto por la lengua?

(Barthes, 2008)

El epígrafe que abre Hacer sapito proviene de Electra, una tragedia de Sófocles (2004) que pone en escena el deseo de venganza de la protagonista por el asesinato de su padre. Su deseo se manifiesta como espera de su her- 
mano Orestes, quien llevará a cabo la venganza, pero fundamentalmente como expresión: de dolor, lamento, injusticia. Es la voz de Electra la que ocupa todas las escenas de la obra y en todas es constantemente condenada al silencio por el resto de los personajes con los que se encuentra: su madre (“¿No guardarás religioso silencio y me dejarás celebrar un sacrificio, ya que te he permitido decir lo que has querido?"), su esperado hermano ("No hablar más de lo que la ocasión exija", "abandona todo discurso vano", "te aconsejo que calles", "no necesitamos ya de más dilatados discursos") y finalmente El Ayo, el anciano que rescató a Orestes, le dice: "Creo que ya hemos hablado bastante, Electra”. En líneas generales, la tragedia de Sófocles se ocupa de los discursos de las mujeres ya que los parlamentos más largos son pronunciados por Electra y luego por su madre, su hermana y el coro (conformado por mujeres). A diferencia de la versión de Eurípides que se concentra en la acción de los hombres, "en Sófocles, lo medular de la obra está concentrado en la heroína que es el espacio de las palabras" (Saravia, 1999). El epígrafe, entonces, es de una tragedia que pone en escena voces de mujeres definidas dentro de la institución familiar: madre, hija, hermana. En Hacer sapito las voces de la hija y del padre, que también están definidas por el marco de lo familiar, funcionan como los personajes dramáticos de una puesta en escena de dos voces que se enfrentan y se configuran: la del padre articula la matriz patriarcal mientras que la de la hija, al contar con ese mismo material, diseñará posibles estrategias para desactivarla.

Si bien es cierto que la libertad lexical de los poemas dialoga con sus contemporáneos de la 18 whiskies, en Viola Fisher los elementos de la oralidad funcionan en otra dirección. En primer lugar, evidenciados por el corte y la brevedad, los versos podrían independizarse del poema, como "objects trouvés discursivos" de estereotipos (en relación a la casa), del discurso médico (en el cordón umbilical que deviene cerebral), del religioso (en el mito de la costilla), de la ley civil y divina (en el casamiento a los ojos de Dios). Los restos de hablas se engarzan como miniaturas, de ahí la brevedad de los versos, que son fragmentos o retazos recolectados en un patchwork discursivo (y en esto podría pensarse un retorno en lo formal de la actividad de tejer, bordar y coser que aparecía como metáfora de la memoria en los poemas de La casa grande de Tamara Kamenszain (1986): 
"Vértigo encimado entre costuras/ hoy está tironeando el parecido/ de la manga que entuba ese pasado"; y también como metáfora de la escritura en los ensayos de El texto silencioso (1983): "Coser, bordar, cocinar, limpiar, cuantas maneras metafóricas de decir escribir”. Pero en Viola Fisher no hay metáfora, la costura se vuelve literal, o mejor, material: parte del proceso de engarce discursivo con que se escriben las voces en los poemas).

La oralidad, además, aparece en fragmentos de canciones infantiles ("qué linda/ manito que tengo/ yo") y frases hechas ("Mi mamá me mima") que usa la voz de la hija, sugiriendo que ese es el material que dispone para nombrarse, configurado por los lugares que asignan los esquemas reguladores que producen las posibilidades inteligibles de los cuerpos. Para Judith Butler (2002) el "sexo" no es una condición original del cuerpo si no un proceso que se desarrolla a través del tiempo mediante la repetición de normas reguladoras que producen la materialidad de ese cuerpo y lo vuelven inteligible dentro del marco del binarismo normativo heterosexual. La performatividad de género y la materialidad del sexo son un proceso conjunto mediante el cual el sujeto se forma y vuelve inteligible un cuerpo que se ofrece como un objeto de producción, control e inscripción de la norma social. Lo que interesa de la propuesta teórica de Butler es el énfasis en que este imperativo, el orden simbólico patriarcal, se realiza a lo largo del tiempo con medios discursivos que habilitan determinadas identidades sexo-genéricas. Advertimos, entonces, que en Hacer sapito el binarismo de los géneros que atraviesa las escenas de infancia y configuración de la identidad aparecerán codificadas, por ejemplo, en "ser Barbi" o "dejarse crecer la barba".

La canción infantil, además de aparecer directamente citada, está en la rima y el ritmo de los poemas, marcado por la métrica de los versos, que comparten brevedad y cantidad de sílabas, la canción infantil retorna como forma para usar la lengua del padre, cuya voz, por ejemplo, dirá: "con la rima me saca/ la lengua y me saca/ de quicio". Así, si por un lado el habla -de la hija- está estructurada como una canción infantil sugiriendo que no tiene la posibilidad de nombrarse por fuera de posiciones hegemónicas ni en otro lugar que el que le asignan los esquemas reguladores que producen las posibilidades inteligibles de los cuerpos (barbi o barba, muñecas o fútbol); por otro, la canción retorna para usar la lengua del padre: "con 
la rima me saca la lengua”, y hacerla fallar. En estos poemas la rima y el ritmo destotalizan, rompen, desunifican, muestran los restos, trabajan con el lenguaje deshecho y con el lenguaje como desecho.

En segundo lugar, a la luz del concepto freudiano de repetición en tanto proceso en el que una fuerza deshace lo que la repetición intenta fijar, advertimos que en Hacer sapito se trabaja con la repetición como falla: casa se repite como casca, barbi se repite como barba. La hija repite "mal" la palabra (y representación) "barbi”: "le dije la quería Barbi/ y ella estúpidamente se dejó crecer la barba". Se trata de una repetición fallida que deja una marca en la escritura, en el cambio de "i" por "a". La repetición da cuenta de lo que falla en el sujeto como totalidad coherente y consistente. En el poemario se articulan repetición y error, fisura o perturbación, como modos de intervención sobre la lógica patriarcal y, en consecuencia, la falla deviene posición discursiva desde donde la voz dramática de la hija se hace, paradójicamente, un lugar de enunciación.

Otra estrategia con que la hija ocupa la lengua del padre es el corte que está tanto tematizado (en: podas, tijeras, gilletes, pedacitos, tajos y bocas que aparecen constantemente) como formalizado en los versos. Se trata de un corte de verso que transforma la morfología de las palabras y la sintaxis de la frase. Sirve de ejemplo lo que ocurre en un fragmento del poema 22 con la palabra "una":

\author{
siendo una \\ equivocación de uno \\ no puede \\ ser feliz nunca más \\ en la vida de esa \\ equivocación que se \\ parece a una niña \\ de su mamá porque \\ de su papá es una \\ cosa errónea [...]
}

Allí, sin el corte, la palabra "una" hubiese funcionado en la frase como artículo ("una equivocación"), pero, por el corte, queda como predicativo 
del verbo ser y se autonomiza, deviene sustantivo, nombre: "siendo una", donde, además, hace audible la exclusión inscripta en la ontología occidental del "uno" universal masculino. Sin embargo, Hacer sapito parece ser menos optimista: esa afirmación y la posibilidad de pensar al ser como "una" en vez, o a la vez, de "uno" es inmediatamente tensionada en el verso siguiente: una es la equivocación del uno, lo que recuerda que el poema exhibe las contradicciones que lleva inscriptas el material con que está hecho. Por citar otro ejemplo, en el verso "Decidiste nacer una / semana antes con el cuerpo", el corte convierte al artículo "una semana" en modificador del objeto directo ("nacer") del verbo "decidir": decidir nacer una (en vez de uno), donde también se visibiliza la decisión como posición activa. Retomando a Adorno: ¿funciona entonces el arte como "lugarteniente de una praxis mejor", por ejemplo aquella en que el ser nombraría indistintamente a una o a uno, o bien se comporta como una máquina que en el intento por ir hacia ese estadío se ha roto y gira sobre sí misma, fallada?

En Hacer sapito, mientras que el cuerpo del padre no es mencionado en todo el poemario (quizás porque, como sostiene Butler (2002), la materia en su forma masculina se presenta como un cuerpo que no es un cuerpo y, en su forma femenina, como una materia que no es ningún cuerpo), el de la hija aparece constantemente como superficie de inscripción de la voz del padre. Así, es constantemente nombrado por partes, fragmentado: boca, manos, paladar, huesos. O bien, directamente tematizado: "y yo tengo en pedacitos/ un cuerpito"; caracterizado desde la falta: "qué pena/ sin pene/ nació", la deformación ("Vos sola/ te mutilaste/ solita nomás/ decidiste nacer una/ semana antes con el cuerpo/ formado a medias/ no quisiste/ esperar el crecimiento/ de los atributos que debe/ un primerizo a su padre no/ podías desilusionarme/ desde el comienzo/ nada entre tus piernas/ inválida") o la malformación ("no es tuya/ la culpa de nacer/ con malformación hijo"). En efecto, en el único poema donde se menciona el cuerpo de la hija en su integridad, aparece partido, literalmente cortado entre un verso y otro, y este corte apunta a reinscribir en la autonomía del cuerpo de la hija, el cuerpo del padre: "Cuer-pito". Sin embargo, la fragmentación que por un lado imposibilita crear una figuración para la hija, por otro es lo que la hace posible. Si en "Cadáveres" el cuerpo aparecía en los pliegues del lenguaje, en el rulo de la repetición sonora: "laz zarigueyaz de dezechoz, 
donde tatúase, o tajéase (o paladea) /un paladar, en tornos/ hay Cadáveres" (Perlongher, 1997); aquí el cuerpo aparece en el corte del lenguaje.

Si en Hacer Sapito el cuerpo femenino emerge como punto de articulación del poder-discurso (Genovese, 1998: 87), el de la soberanía masculina y patriarcal sobre la palabra, el corte es el acto que abre la posibilidad de la escritura (por eso las hojas para escribir devienen hojas de afeitar y se escribe con una gillete en la mano); allí un cuerpo propio e impropio se expone $-\mathrm{y}$ habla - por medio de la fragmentación de la palabra del otro. En el tercer poema la hija dice: "De mamá tuve un cordón umbilical / y de papá también / tuve un cordón/ cerebral..." el cual termina: "y mi boca es un tajo/ al nudo/ atroz un tajo y sangre/ como las manos de papá/ intentando anudar/ otra vez su cordón". Es en el corte y la falta (o bien en la ausencia, el vacío o el espacio en blanco: por eso la hija "nota el silencio en cada frase") que podrá aparecer un espacio para la voz de la hija y, quizás, un nuevo nombre. En este punto, la escritura de Viola Fisher puede pensarse a la luz del pensamiento de Nancy (2003) sobre la escritura del cuerpo como excripción: un "hablar-cuerpo" en tanto acontecimiento que expone la fractura de sentido, donde la escritura "sólo vale directamente en tensión sobre el sistema significante” y el signo hace cortes al discurso. Las estrategias de corte y repetición funcionan en el poemario sobre dos tópicos que quiero comentar brevemente: la casa y la patria.

\section{Ni un cuarto propio ni una casa entera}

En cada signo duerme este monstruo: un estereotipo

(Barthes, 2004)

Lo doméstico es universal en letra de varón, y en letra de mujer es personal, íntimo: no es político

(Marina Mariasch, 2017)

Para Barthes la lengua es "simplemente fascista" porque obliga a decir. Viola Fisher escribe con una lengua que la obliga a decir, pero allí aparece 
el corte de verso y hacia dentro de las palabras y la repetición como falla. En la serie de poemas hay tres poemas en los que aparece la palabra "casa". Pero ¿̇se trata del escondite doméstico? ¿El lugar de lo íntimo, lo privado, la familia?

Mi casa es una entera casa miento está rota y negra como los ojos de

Dios la hizo partida en cuatro ojos dos hijos velan.

En el reparto patriarcal de los cuerpos en el espacio, lo doméstico corresponde a la mujer: la "reina del hogar", el "ama de casa". Viola Fisher se hace cargo del estereotipo y de la casa como tópico de lo femenino, por eso el primer verso del primer poema afirma el reparto que une a la mujer con el hogar, pero inmediatamente, en el segundo verso, lo contradice. La casa miente al nombrar el lugar de pertenencia ("mi”), por eso al decir "Mi casa": "miento". En la casa, no tiene "un cuarto propio" donde escribir, si escribe es más bien porque está rota: son los restos de "la casa" los que permiten hacerlo. En el siguiente poema sobre la casa aparece la repetición como falla, que ya señalamos en otros poemas:

Mi casa es una entera

casca

miento porque al quebrarse

estaba llena de jugo

podrido y casca

es hollejo

solamente corteza

de las uvas se hace

el vino de mi casa

es sangre. 
La casa se hace casca, corteza quebrada, y son esos restos materiales los que proliferan en este poema en las aliteraciones de consonantes; sonidos de quebraduras: casa, casca, porque, quebrarse, podrido, corteza.

El trabajo de este poemario sobre el tópico de lo doméstico, la casa, la familia como aquello que sería propio de "lo femenino" no es de "reinterpretación", no se lo apropia para darle un nuevo sentido, sino de vaciamiento, para dejarlo simplemente expuesto. Así, los dos últimos versos exponen mediante la yuxtaposición la violencia que duerme en el estereotipo "el vino de la casa".

Como ya señalamos, es cierto que Hacer sapito aparece en un momento en que en la poesía argentina ya existían varios textos publicados escritos por mujeres. Para ese momento ya estaban consolidadas las obras de poetas que venían publicando al menos desde principios de los 8o, por ejemplo, Diana Bellesi explora el deseo y la erótica por fuera del marco heterosexual, y Tamara Kamenszain, quien en La casa grande (1986) y Vida de living (1991) da otro sentido al universo doméstico que emerge como espacio creativo, ligado al dominio intelectual de la artesanía y el saber más que a la irracionalidad y el sentimentalismo (Genovese, 1998: 87). Allí la casa deviene, además, un espacio-tiempo de capas familiares significantes donde emprender una búsqueda del origen. Alicia Genovese (1998) analiza también las obras La luz en la ventana (1982) y El mundo incompleto (1987) de Irene Gruss, donde advierte que el espacio del hogar se convierte en espacio de resistencia por una voz que desde ese espacio reflexiona sobre la historia y resemantiza el rol tradicional de mujer ama de casa. Pero Viola Fisher se distancia nuevamente de esta serie ya que el espacio de la casa no es íntimo, privado, individual, no es el lugar donde la mujer, encarnada en este poemario en la voz de la hija, encuentra una voz, tampoco es donde transcurre un linaje femenino en el que habría un saber que se pasa de abuelas a madres sino que, como venimos analizando, es el espacio de una disputa y está atravesado por la violencia. De este modo, Viola Fisher expone a lo privado, la casa, como político. La casa y la lengua aparecen como espacio público, ese espacio privado que deviene público, movimiento encarnado en el desplazamiento de casa a casca o de mi casa a miento. Verónica Viola Fisher cuestiona roles tradicionales de género y sexualidad e interpela el control patriarcal del espacio público y del privado o "íntimo" al mostrarlo también como público. 


\section{Pater, Patria, Palabra}

Que no se puede eliminar del arte las tensiones de las que surge

(Adorno, 2004)

aunque estuviera completa en mi apellido / no sería yo entera, algo me han quitado / Cuando nací / y hasta cuando fui concebida, en mi país / en mi lengua

(Viola Fisher, 2005)

Cae por la izquierda un pesado cortinado rojo que, atado por el centro, deja ver por detrás un paisaje, el cual ocupa el tercio superior de la tela. Sin embargo, lo que se destaca es la figura, quizás por su iluminación, enfática, definida y uniforme: el padre, sentado en un sillón de madera labrada, toma con firmeza a dos niñas paraditas a su lado, una apoyada en cada una de sus piernas. Solo una de ellas nos está mirando pero lo que más llama la atención son sus rostros adultos, parecen señoras miniaturizadas con vestiditos de volados exagerados. La extrañeza de esos rostros en esos cuerpos hace que la mirada avance por partes, haciendo recortes. Al lado de los rostros de estas niñas-viejas, el pintor Valdassare Berassi ha ubicado las manos que podrían ser puntos imaginarios de una diagonal que atraviesa el cuadro. Las manos del padre tienen un gesto firme: la izquierda toma la mano de una de las hijas mientras que la derecha se apoya en el hombro de la otra, a modo de abrazo. Pero también, cada una de las hijas, como si el sol estuviese justo sobre sus cuerpos, proyecta sombra sobre las manos paternas. 


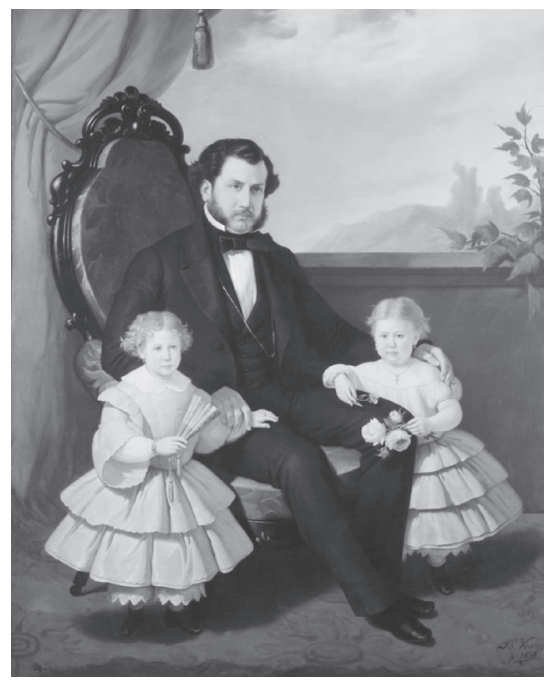

Se trata del "Retrato de caballero y niñas", pintado en 1858 por un pintor italiano que vino a Buenos Aires con la moda de los retratos, a trabajar para familias patricias... y algo de este cuadro hace pensar en Hacer sapito. ¿Qué tiene este cuadro de mediados del siglo XIX en cuya trama retorna, anacrónicamente, el poemario de 1995?

Aquella época puede caracterizarse como el proceso de formación de los Estados Nación, en el cual la trágica Guerra del Paraguay funcionó como emblema de la ficción nacionalista y como excusa para fijar los límites territoriales. La patria, como figura de la utopía moderna que en América Latina se hace efectiva por "la gesta heroica" de "los padres de la patria", se consolida, podríamos decir, junto a la figura del prócer y al significante "padre". El padre como emblema de la patria, o bien, la voz del pater entremezclada con el emblema de la patria: por eso el himno nacional es otro material con el que trabaja Hacer Sapito, en su tercer poema.

Es igual a la madre que

no es madre es llenadora

de cabezas huecas

con mierda hay que limpiarles

la boca

antes de hablar 
de mí porque soy yo

el único

que supo conseguir, los laureles

y las hará jurar

con gloria

conglomerado de conchas

vos, tu madre, tu abuela

mi futura nieta seguir

calladas.

Como también ha señalado Marina Yuszczuk (2011), en este poema la voz del padre se funde con la voz de la patria, ya que aquel habla mediante el himno nacional ("el único/ que supo conseguir, los laureles/ y las hará jurar/ con gloria”). Entonces, por un lado, el trabajo con el himno en este poema traza un vínculo con aquella reescritura de Leónidas Lamborghini, "SEOL" (en efecto, reeditado en 1996, un año después de la publicación de Hacer sapito), en el cual digiere la tradición y subvierte estructuras para reinstalar la pregunta por la identidad. Pero mientras que Lamborghini se circunscribiría a la pregunta por lo nacional, Viola Fisher introduce allí, en las grietas de "lo roto de la identidad", el interrogante por la voz de la mujer (y por la mujer) en esa nación y tradición. El pater es entonces representante de la patria (pues el himno habla de él: "de mí porque soy yo") y con el patrimonio de la palabra ordena el silencio de las mujeres de la familia, en otra escena atravesada por la violencia: "vos, tu madre, tu abuela/ mi futura nieta seguir/ calladas”. (En estos versos también podríamos escuchar la resonancia de aquel verso de Neruda "me gusta cuando callas/ porque estás como ausente", una de las construcciones de la mujer que como metáfora y objeto en el discurso literario, dominado por el discurso patriarcal, aparece como fetiche amoroso y amada inmóvil, caracterizada principalmente por el silencio y la ausencia).

Allí, frente a esta orden, el poemario procede con el corte. Lo que muestra son ruinas de determinadas construcciones y representaciones de la casa y de la patria. La poeta Marina Mariasch (2016) reflexiona: "La femineidad pareciera todavía que está mal vista. Por un lado están esos casos que más de una vez leímos: artículos o tuits donde la que escribía se disculpaba o aclaraba odiar "todo lo minita”. Ejemplos como que se adjetive 
con acepciones como "muy minita" y demás significantes aplicables solo al género femenino, son un índice transparente de que en la arena del lenguaje, donde se juegan las luchas históricas, algo sigue igual a pesar del largo camino de logros conseguidos". Podríamos decir que en Viola Fisher una escritura "de minita" es una escritura minada. Si el material que posee son las palabras del padre (sus posiciones, relatos y posibles), la lengua deviene campo de batalla y conjunto de ruinas o, parafraseando a Rancière (2012), la lengua se muestra como un común litigioso: allí donde los seres parlantes disputan su existencia y derecho a la palabra. Con los encabalgamientos se quiebra el orden de ese estado de lengua, se interviene una sintaxis de la frase. Si el corte funciona como motor de escritura, los poemas se forman sobre una lengua en ruinas, que se ve en la brevedad de los versos.

\section{Sobre las ruinas del nombre del padre}

algunas de nosotras continuamos recurriendo al saqueo del Logos a causa de la utilidad de sus restos

(Butler, 2002)

Cuando dije la palabra / papá dije pupa

(Viola Fisher, 2005)

¿Cómo disputar la inteligibilidad del cuerpo al marco patriarcal? ¿Cómo escribir con otra palabra a la del padre? ¿Cuál es el lugar de la hija por fuera de la voz del padre? ¿Cuál es la enunciación por fuera de la institución familiar? ¿Cómo nombrarse si no es hija, nieta, madre, abuela o niña? ¿Hay un afuera de ese estado de lengua en Hacer sapito? El último poema pareciera cerrar cualquier afuera: aun antes de nacer, en el nombre, ya está inscripta la violencia. Este poema final excede la serie del nacimiento de la hija a la muerte padre y amplía el espectro de producción de los cuerpos: antes de nacer, en el nombre, ya está inscripta la violencia, la amputación, la falta, recordando las ideas de Butler sobre la materialidad del cuerpo en Cuerpos que importan: no hay cuerpo previo al signo y "la materia misma 
está fundada en una serie de violaciones" (Butler 2002: 56), en efecto, no hay materialidad que no sea efecto del poder. Por este motivo es que en Viola Fisher todo es público, sea el cordón umbilical, el cordón cerebral, la casa, la familia, la infancia.

Sin embargo, si bien no hay un estadio de la materia previo al lenguaje (porque antes de nacer ya tenía un nombre), lo que el poemario sugiere es que cierta dimensión impersonal, una materia que rebasa -ahora sí- el marco butleriano, se alcanza posteriormente: después del cuerpo, después de la materia, después del lenguaje. Es hacia una zona orgánica mínima que tiende la voz de la hija, luego de 45 poemas en los que procesa -tajea, corta, poda, saquea, fragmenta, desplaza, hace fallar- la palabra del padre.

muerta de padre

me protegen los gusanos de la tierra

Muerta de padre, que en estos poemas es también muerta de palabra, la voz de la hija encuentra un espacio entre los gusanos de la tierra, es llamativo también el contraste entre la violencia que atraviesa las escenas de infancia y configuración identidad de todos los poemas con este nuevo vínculo de cuidado y protección. Todo el poemario, entonces, tiende hacia ese umbral: desde la identidad de la hija como "hija" a una dimensión orgánica donde el libro termina (...esa dimensión en la que sí se desplegó la escritura de Clarice Lispector, por ejemplo, en Agua Viva). En esa dimensión esta voz parece encontrar lazos con formas de vida mínimas, pero no desarrolla aun una lengua. Para este poemario, lo que excede la lengua del padre no es inteligible. Entonces, si bien no tiene palabras permanece en la tierra y aquí la tierra también puede leerse como lo que desmiente al territorio de la patria. Esta dimensión ya se anunciaba en el comienzo del libro en uno de sus epígrafes, el que cité al comienzo de este apartado: la palabra papá se dice (en su versión fallida) pupa. Si vamos al significado de pupa, hallamos que puede ser: llaga supurante en la boca; estado de metamorfosis de los insectos entre la larva y el adulto que en enciclopedias de zoología y biología se describe como "aparentemente detenido" o estado amorfo del cuerpo del insecto. En su primer sentido, refiere a la boca que hace fallar la lengua del padre, quebrándola y haciéndola supurar, volviéndola fluida (la serie de la sangre es elocuente). En su segundo sentido, este poemario 
nos deja la pregunta sobre los poemas que serán escritos en la lengua de los gusanos de la tierra.

\section{Referencias}

Adorno, Theodor. (2004). Teoría estética. Akal: Buenos Aires.

Arnés, Laura. (2016). Ficciones lesbianas, Buenos Aires: Madreselva.

Barthes, Roland. (2004). El placer del texto y lección inaugural, Buenos Aires: Siglo XXI. p. 118.

Barthes, Roland. (2008). Fragmentos de un discurso amoroso, Buenos Aires: Siglo XXI.

Bellessi, Diana. (1988). “La diferencia viva”. Diario de poesía, 9, 9.

Butler, Judith. (2002). Cuerpos que importan, Barcelona: Paidós.

Gambarotta, Martín. (2006). "El habla como materia prima”, Tres décadas de poesía argentina 1976-2006. Buenos Aires: Libros del Rojas.

Genovese, Alicia. (1998). La doble voz. Poetas argentinas contemporáneas. Eudeba: Buenos Aires.

Gruss, Irene. (2008). Obra poética reunida 1982-2007. Bajo la luna: Buenos Aires.

Kamenszain, Tamara. (1983) El texto silencioso. Tradición y vanguardia en la poesía sudamericana. México: UNAM.

Kamenszain, Tamara. (1986). La casa grande. Sudamericana: Buenos Aires.

Longoni, Ana. (2014). Perder la forma humana. Una imagen sísmica de los años ochenta en América Latina, Buenos Aires: UNTREF.

Mariasch, Marina. (2016). "Poesía y género”, texto leído en el I Encuentro de Poesía y Género. Disponible en: https://poetasperonistas.wordpress.com/2016/o7/12/poesia-y-genero-por-marina-mariasch/

Mariasch, Marina. (2017). "Machismo y literatura. El mercado de la sensibilidad”. Anfibia. Disponible en: http://www.revistaanfibia.com/ensayo/machismo-y-literatura-el-mercado-de-la-sensibilidad/

Nancy, Jean-Luc. (2003). Corpus, Madrid: Arena.

Perlongher, Néstor. (1997). Poemas completos. Buenos Aires: Seix Barral. Porrúa, Ana. (2011). Caligrafía tonal. Buenos Aires: Entropía.

Rancière, Jacques. (2012). El desacuerdo. Buenos Aires: Nueva Visión. 
Rubio, Alejandro. (1997). "Otra patada al gusto”. Diario de poesía, 44, 3537.

Salgado, María. (2016). Hacía un ruido. Frases para un film político, Madrid: Contrabando.

Saravia de Grossi, María Inés. (1999). "Electra de Sófocles: Una interpretación”, Synthesis, 6, 99-114. Disponible en: http://www.memoria.fahce. unlp.edu.ar/art_revistas/pr.2736/pr.2736.pdf

Sófocles (2004). Electra. Ayax. Ediciones Libertador: Buenos Aires.

Viola Fisher, Verónica (2005). Hacer sapito, Buenos Aires: Gog y Magog. Yuszczuk, Marina (2011). Lecturas de la tradición en la poesía argentina de los noventa (tesis de posgrado), La Plata: Facultad de Humanidades y Ciencias de la Educación, Universidad de La Plata. Disponible en: http://www.memoria.fahce.unlp.edu.ar/tesis/te.742/te.742.pdf 